\title{
PRODUCT/SERVICES INFORMATION
}

- TopoMetrix introduces the TMX Scanning Probe Microscope. Allowing high resolution, three dimensional images of objects as small as atoms, the TMX 2000 family allows three initial imaging modes - SFM, STM and LSTM. Exclusive features include integrated vibration isolation, optical microscope and an automated sample positioner. TopoMetrix, 1505 Wyatt Drive, Santa Clara, CA 95054. Tel: (408)982-9700, Fax (408)9829751.

- NORAN INSTRUMENTS, Inc. guarantees Beryllium detection using an 1Atmosphere UTW with the Pioneer Series $S i(L i) X$-ray detector. NORAN Instruments, Inc. (608)831-6511.

- Magnification reference standards for optical and scanning electron microscopes are available from Geller MicroAnalytical Laboratory. Calibration range is from $10 \mathrm{X}$ to $50,000 x$. The unique pattern permits calibration in both $X$ and $Y$ axes, measures distortion, and determines recording CRT performance. NIST traceable. Geller MicroAnalytical Laboratory, One Intercontinental Way, Peabody, MA 01960. (508)5355595.
- National Institute of Standards and Technology announces the NIST/NIH Desktop Spectrum Analyzer Program and X-Ray Database. This computer program, for the Macintosh $P C$, performs rapid yet detailed generation, interpretation, and analysis of X-ray spectra. It emulates the specimen properties and experimental environment to generate spectra reflecting the relevant physics, chemistry, and statistics of a specimen under electron bombardment. It provides all of the usual MCA features. For further information or to order, call (301)975-2208.

\section{- E. Fjeld, an established manu-} facturer of custom accessories for electron microscopes, now offers high precision specimen stages for SEMs - including Modular Plus with $100 \mathrm{~mm}$ continuous $Z$ motion and complete stage motorization. E. Fjeld Co., 3 Executive Park Drive, North BilLerica, MA 01862. Tel:(508) 667-1416.
- Now avai lable from AMRAY, a full line of field emission scanning electron microscopes. With a new, state of the art, Schottky field emission gun, AMRAY offers the most versatile field emission SEMs in the industry. For further information on our field emission SEMs, tungsten or LaB6 models, please write to AMRAY, 160 Middlesex Turnpike, Bedford, MA 01730 or call (617)2751400.

- Analytical reference standards for microanalysis are offered by Geller MicroAnalytical Laboratory. Over 200 different elements, compounds, glasses, and alloys are available for the ultimate in measurement accuracy. Custom built. Typical delivery time is 2 weeks. Geller MicroAnalytical Laboratory, One Intercont inental Way, Peabody MA 01960. (508)535-5595.

\section{POSITIONS AVAILABLE}

Advertisers may elect to run "blind" employment advertisements, in which case a Microscopy Today box number will be used for responses. The responses, in turn, will be forwarded promptly to the advertiser. Respondents to "blind" advertisements may indicate which companies (i.e. their current employers) that are not to receive copy of their resumes.

- Major, expanding, independent testing lab in Eastern U.S. is seeking a senior member of management. Responsibilities include being completely knowledgeable in all techniques relating to materials analysis and to present capabilities and results to clients. Ideal candidate would have a PhD typically in geochemistry, mineralogy, material science, etc. Outstanding analytical, interpretive and communication skills are required. The ability to manage a group of technologists, and to present technical papers, would be clear pluses. Excellent compensation plan. Respond to Microscopy Today. PO Box 122-AA.
- Pinchin Harris Holland Associates, Inc., Seattle WA, is seeking a qualified electron microscopist to manage and maintain their asbestos analysis Lab. A minimum of three years experience in TEM asbestos analys is and a Bachelors degree in geology, materials science, biology or other science is desired. Experience in zone axis identification of diffraction patterns and AHERA methodology a must. Image analysis and computerized applications experience desirable. Contact John Harris by FAX: (206) 789-8424.
- Job opportunity for TEM operator. Material science background, BS and up preferred. Contact Frances Hice before 12 Feb. US Bureau of Mines, Albany, OR. (503) 967-5837. 